\title{
Application of Chaotic Genetic Algorithm and Elman to Assess Flight Per- formance Under Multiple Physiological Signals
}

\author{
Wu Jingjing, Yang Zheng, Wu Qi and Fu Shan*
}

School of Shanghai Jiaotong University, Shanghai, 200240, P.R. China

\begin{abstract}
In the recent years, the assessment and forecasting of flight performance based on pilot's multiple physiological parameters has become an important theme of research. However, traditional forecasting and assessment of flight performance is mainly based on the manual assessment or explicit mathematical models, and rarely take the physiological parameters into consideration. Based on the complex structure with multi-dimension, nonlinearity and information-related to physiological parameters, a hybrid model based on chaotic genetic algorithm and Elman neural network (CGAE) is proposed in this paper. We optimize the weights and thresholds of Elman by chaotic genetic algorithms (CGA) and demonstrate that the CGAE hybrid model is well suited for the assessment of flight performance through experiments. Moreover, GA is also adopted to optimize the Elman neural network (SGAE). Experiments also show that CGAE have better predication accuracy and convergence rate than SGAE and Elman, which is indicated that CGA-Elman network has the great application prospect in the field of the assessment of flight performance.
\end{abstract}

Keywords: Chaotic genetic algorithm, Elman network, flight performance, multiple physiological parameters.

\section{INTRODUCTION}

Safety is one of the vital elements of civil aviation. With the fast development of the technology and management, the number of accidents caused by mechanical failure decreased significantly. Human factors become more and more leading cause of accidents. An important aspect of human factor is assessment of a pilot's workload. Piloting an aircraft is a complex task that places demands on several aspects of a pilot's cognitive capabilities [1]. Either excess workload or under-load can have an impact on flight performance. Up to now, many researchers have assessed pilot's workload though multiple physiological parameters [1-3]. Yung-Hui Lee indicated that the pilot's workload could be assessed by the performance and the assessment of flight performance could be defined by the consequence of landing roughly or the deviation of flight path [3]. Thus it can be seen that there exists some relationships between the flight performance and the pilot's physiological features.

Up to now, many researchers have assessed pilot's workload though multiple physiological parameters [4-6]. YungHui Lee indicated that the pilot's workload could be assessed by the performance and the assessment of flight performance could be defined by the consequence of landing roughly or the deviation of flight path [6]. Thus it can be seen that there exists some relationships between the flight performance and the pilot's physiological features.

Multiple physiological signals are characterized by nonlinear, high dimension, complex structure and information.
GA as a representative of evolutionary computation is a direct random search algorithm. Due to its flexibility, versatility and robustness, it shows superiority in optimizing problems of multidimensional and complex data. However, there are some defects on GA. For example, the optimization may get stuck at a local optimum and it tends to converge prematurely. In order to avoid these overcome, it is necessary to adopt some improvements on GA to speed up the convergence and heighten the effectiveness of GA. The chaotic optimization method based chaos theory has come into use as a novel type of random search algorithm during the past decade. Chaos often exists in nonlinear systems. It is the highly unstable motion of deterministic systems in finite phase space [7]. Taking advantage of the universality, randomicity and sensitivity dependence on initial conditions, it is more likely to acquire the global optimum solution. Thus, the combination of chaotic mapping and GA become an effective optimization approach to solve the concerning problems [7-9]. Elman neural network, as one of the artificial neural network, adds a context layer in the hidden layer as a delay operator to store internal states, which make the system a dynamic time-varying capacity and strong global stability. Use chaotic genetic algorithm to optimize the Elman neural network has not been studied in the filed of assessing the flight performance yet. For that sake, this paper is focused on the hybrid model by means of the integration of chaotic mapping, genetic algorithm and Elman network.

By combining the chaotic genetic algorithm with Elman neural network, here we put forward a new chaotic genetic Elman, called CGAE. Based on the CGAE, an evaluation model for flight performance is proposed and verified the effectiveness by experiments. The rest of this paper is organized as follows. Elman and genetic algorithm are introduced briefly in Section 2 and Chaotic Genetic algorithm 


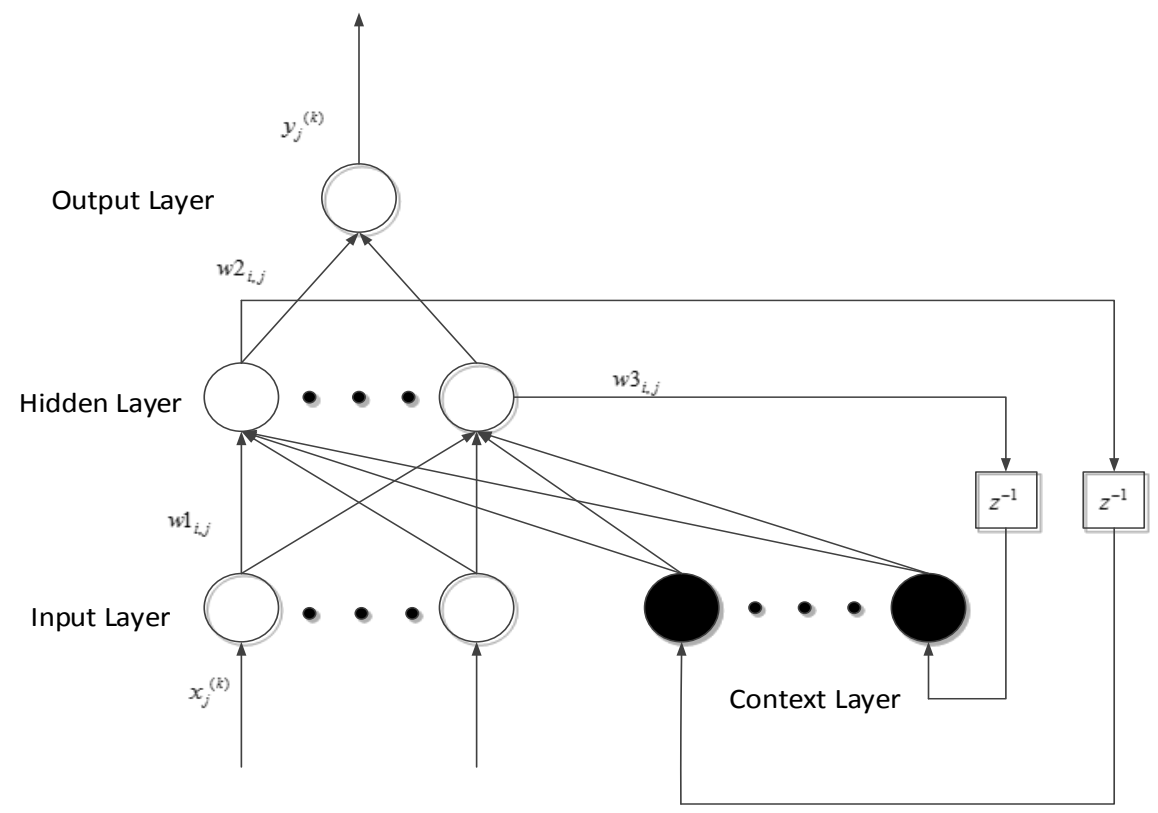

Fig. (1). Structure of elman neural network model.

(CGA) is proposed in this section. Section 3 presents the simulation results and discussion. The final conclusion is drawn in Section 4.

\section{THE HYBRID MODEL OF CGA-BASED ELMAN}

\subsection{EIman Neural Network}

The Elman neural network is one kind of globally feedforward locally recurrent network model proposed by Elman [10]. The structure of an Elman neural network is illustrated in Fig. (1). $z^{-1}$ is a step delay operator. It is obvious that the Elman network consists of four layers: input layer, hidden layer, context layer, and output layer. There are adjustable weights connecting every two adjacent layers. Context layers are used to store the internal states.

The training algorithm for the Elman neural network is based on the gradient descent principle, similar to the BP learning algorithm. From Fig. (1), at iteration $k_{\text {we have the }}$ following relationship:

$o_{-} c_{i}^{(k)}=n e t c_{i}^{(k)}$

where ${ }^{O_{-}} c_{i}$ and ${ }^{n e t}-c_{i}$ are the output and input of context node $i$, respectively. Note that the node activation function of the context nodes is linear. To calculate the input as well as output of hidden node $j$, we have:

$$
\begin{aligned}
& n e t_{-} h_{j}^{(k)}=\sum_{i=1}^{m} w 1_{i, j} x_{i}^{(k)}+\sum_{i=1}^{l} w 3_{i, j} o_{-} c_{i}^{(k)} \\
& o_{-} h_{j}^{(k)}=a\left(n e t_{-} h_{j}^{(k)}\right)
\end{aligned}
$$

$x_{i}^{(k)}$ is the input of Elman neural network, where

$i=1,2, \ldots, m$. net $h_{i}$ is the input of node $i$ in the hidden layer, and $o_{-} h_{i}$ is the output of node $i$ in the hidden layer, where $i=1,2, \ldots l$. The output of node $j$ in the output layer, $y_{j}^{(k)}$, is given by:

$y_{j}^{(k)}=\sum_{i=1}^{l} w 2_{i, j} o_{-} h_{i}^{k}$

where $j=1,2, \ldots, n$. The output of context node $i$ at the $k$ th training iteration is one-step-delayed output of the corresponding hidden node $i$ :

$n e t_{-} c_{i}^{(k)}=o_{-} c_{i}^{(k-1)}$

We can see that the context weights play an important role in the error back-propagation procedure. However, local minimal caused by the regular BP learning algorithm often result in an unavoidably large approximation error that may reduce its prediction accuracy. Integration the chaotic genetic algorithm with the Elman network is an alternative solution to this problem, which is to be introduced in the following sections.

\subsection{Genetic Algorithm}

GA is a learning mechanism of natural selection and evolution. It is a highly parallel, randomized and adaptive search algorithm, which uses the group search technology with population as a solution. Based on the survival and reproduction of the fitness, GA continually exploits new and better solution without any pre-assumption. GA evolves a 


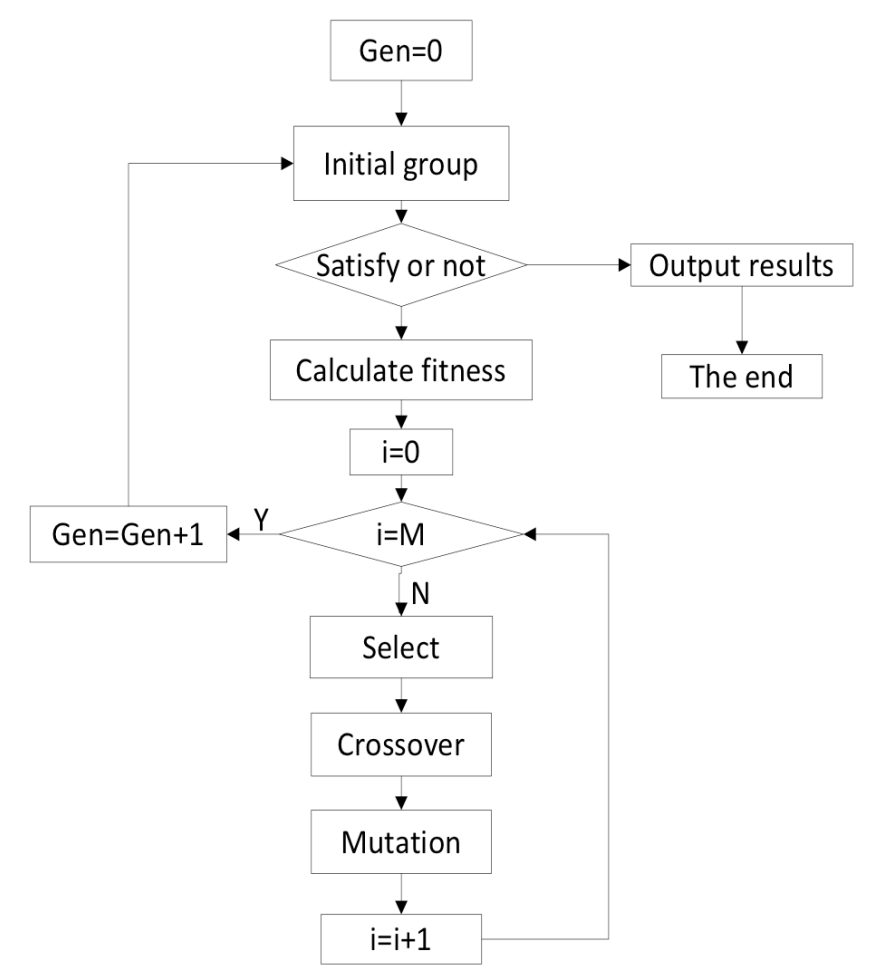

Fig. (2). The flowchart of GA.

population of candidate solutions and each solution is represented by a string called chromosome, which is usually coded as a binary string. GA actually is an iterative process; in each iteration, it retains a candidate solution and sorts their pros and cons, then choose some of the solution according some indicators and use genetic operators computing to produce a new generation of candidate solutions, repeat this process until meet the target convergence [11]. Firstly, individual is encoded by real numbers and every individual is represented by a chromosome. Then, the fitness of each individual is calculated. Following these, individuals evolve towards an optimal solution over successive generations via selection, crossover, and mutation processes. The flowchart of Genetic Algorithm is shown in Fig. (2).

\subsection{Implementation Steps of Chaotic Genetic Algorithm}

Chaos often exists in nonlinear systems. Chaos is not disorderly and unsystematic, but a phenomenon that has a delicate internal structure. It is the highly unstable motion of deterministic systems in finite phase space, which characterized by randomicity, ergodicity and regularity. Many chaotic mappings in the literature possess certainty, ergodicity and stochastic property. In the recent years, chaotic theory have been used to improve the performance of evolutionary algorithm $[12,13]$.

The most well-known chaotic mapping is Logistic mapping, whose equation is [14]:

$$
X_{n+1}=\mu X_{n}\left(1-X_{n}\right)
$$

in which $\mu$ is a control parameter, $n=0,1,2 \ldots$, and $x$ is a stochastic variable. The behavior of system Equation (6) is greatly changed with the variation of $\mu$, i.e. when $\mu=4$, the equation is changed to:

$X_{n+1}=4 X_{n}\left(1-X_{n}\right)$

Thus, very small difference in the initial value of $x$ causes large difference in its long-time behavior, which is the basic characteristic of chaos.

In this paper, we use Logistic mapping to initialize the population for improving the performance of optimization. The initial group of genetic algorithm is generated by Equation (7).

\subsection{CGA-Based Optimization on Elman}

Genetic algorithms have been used in three main tasks: training the weights of connections, designing the structure of a network and finding an optimal learning rule. In this paper, CGA is used to train the weights and threshold of the Elman neural network (ENN). The structure of ENN has been decided firstly. CGA-Elman optimization can be seen as an adaptive system without human intervention and automatically adjust its connection weight and threshold, which realizes the integration of CGA and Elman and is shown in Fig. (3).

In briefly, the basic steps of the proposed hybrid model are as following:

1. Initialize the population. Individual is encoded by real numbers and every individual is represented by a chromosome. The initial weights and thresholds are encoded by CGA, generating the initial population composed by a number of chromosomes. 


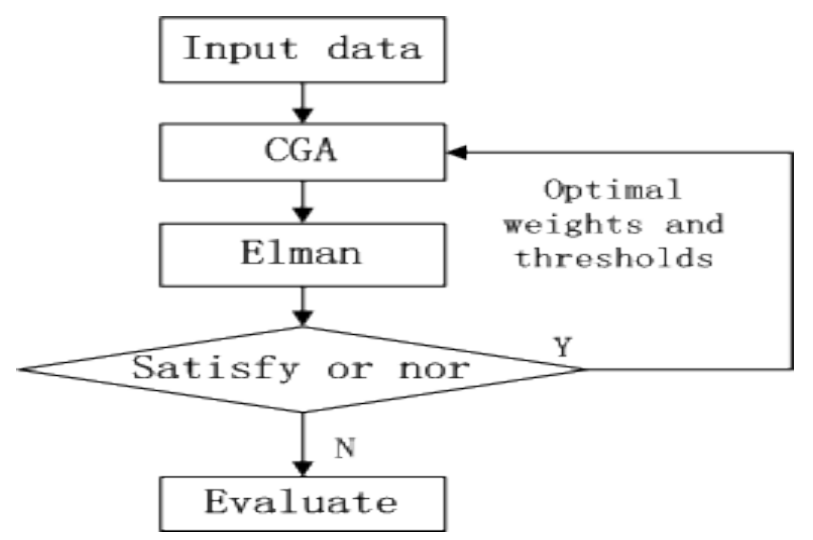

Fig. (3). The structure of CGA-Elman.

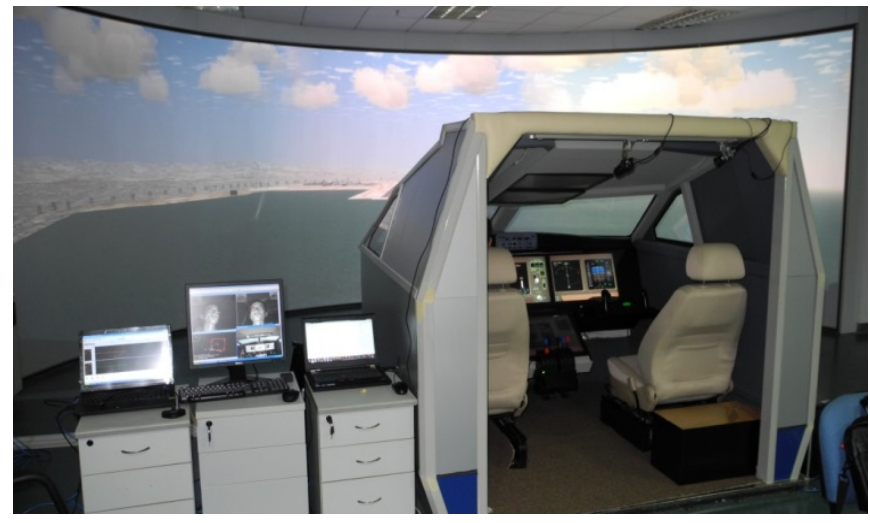

Fig. (4). The flight simulator and monitoring system.

2. Evaluate the fitness. Evaluate the fitness of each chromosome. Forecasting problem is to established a network model which make the forecast error minimum, so the MSE is usually used for this purpose .It formula is described by:

$$
M S E=\frac{1}{N} \sum_{i=1}^{N}\left(y_{i}-\hat{y}_{i}\right)^{2}
$$

where $y_{i}$ is the real value from the testing set, $\hat{y}_{i}$ is the forecasting value. $N$ is the length of the testing sample.

3. Selection. Based on the fitness function, chromosomes with lower fitness values are more likely to be chosen. The roulette wheel selection principle is applied to choose chromosomes for reproduction.

4. Crossover operations. In crossovers, chromosomes are paired with randomly and set a crossover-point in the individual string. Then interchange the part of chromosome and get a new individual.

5. Mutation. Whether the current chromosome is mutated depends on the mutation probability and the position of mutation is randomly confirmed.

6. Stop condition. If the number of generations reached to a given scale, then the best chromosomes are presented as a solution; otherwise, go back to Step 2.
7. Train the Elman network using the optimal weights and thresholds. The genes on the best chromosome represent the optimal weights and thresholds which set as the initial values of weights and threshold. Then calculate the error of the network.

8. When reaching the largest training epochs, the training is ended.

\section{EXPERIMENT AND DISCUSSION}

\subsection{The Data Description}

The CGA-Elman is used to solve the matter of the evaluation of flight performance via multiple psychophysiological parameters. The apparatus of this experiment is comprised of two parts: the Boeing 777-200ER flight simulator and the physiology monitoring system. The flight simulator, as shown in Fig. (4), was built for simulated flight. The flight path is set by the open software, FlightGear. Psycho-physiological devices are the Smarteye 5.8 eye tracker device and the BioHarness physiological parameters monitoring equipment (wore by subjects to record the heart rate data and respiration data). The instantaneous variations of some physiological measurements can be seen on the computer, as depicted in Fig. (5). The flight data, such as airspeed, altitude and acceleration, are also recorded by FightGear. 

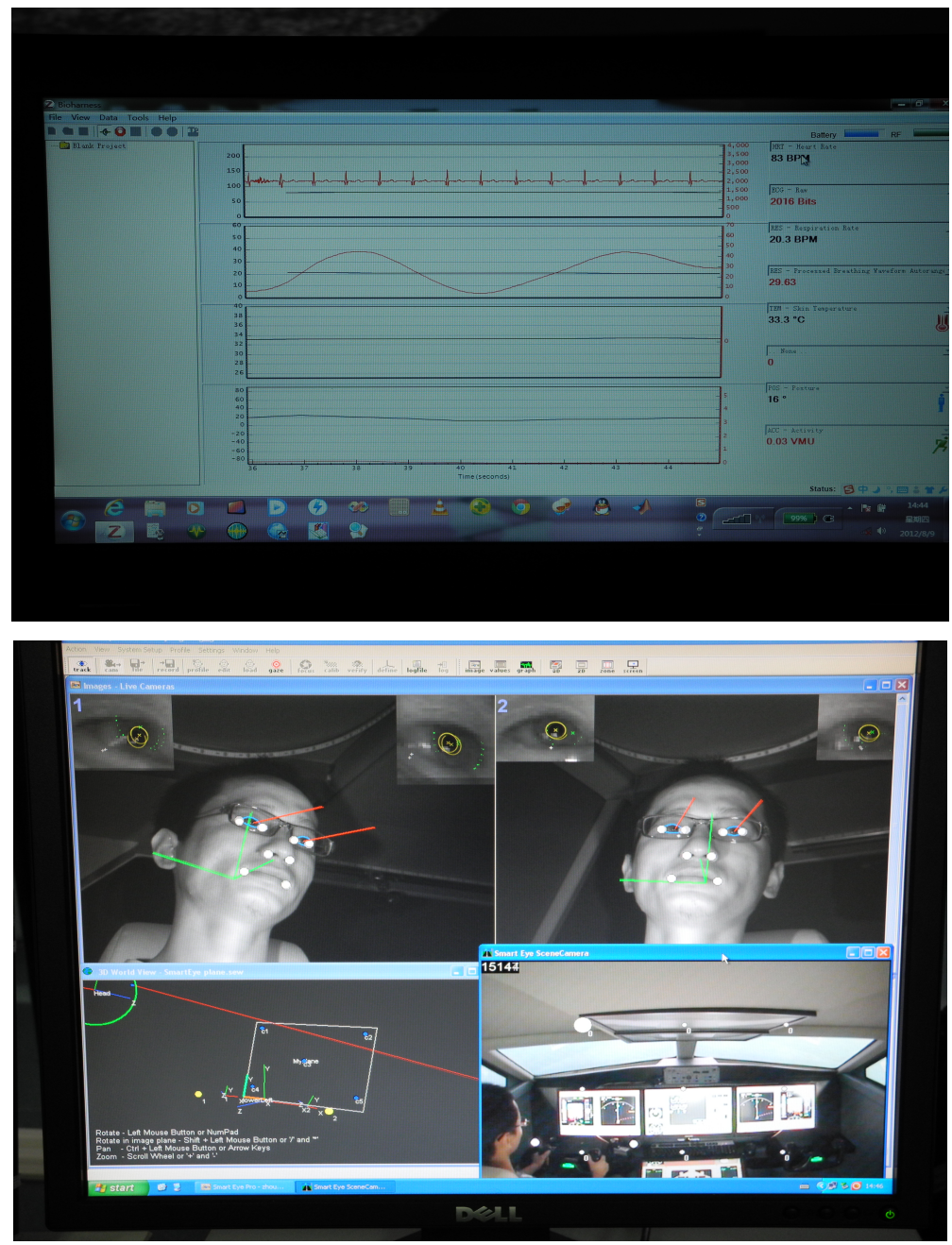

Fig. (5). Variations of physiological measures when flight.

Because the approach and landing phase is the process of the highest accidents rate, which require pilots to concentrate highly on all kinds of information and to manipulate the aircraft frequently, the performance of flight will affect the aviation safety directly. Four novice pilots were invited to perform the planned flight mission: approach and landing. During the process, subjects need to pilot the aircraft according to the glide slope, extend flaps 5 times at the given altitude based on instructions as well as controlling the airspeed. Each subject performed 5 times. So the total time of flight is 20 and we got 20 sets of data. The whole process lasts 3-4 minutes. According to the past papers $[1-4,15]$, physiological parameters and eye movement data were chosen as following: Heart Rate (HR), Respiration Amplitude (Res_Amp) and Respiration Rate (Res_Rate), Eye Blink Interval (BI), Eye Fixation Time (FT). According to the definition of Yung-Hui Lee in his paper [6], the performance in this paper is represented by flight path deviations.

The original data was sampled at different frequency from the devices and become the experimental data sets through two sampling. All the values were preprocessed and normalized. We use min-max normalization method, which transforms the data into the desired range $[0,1]$. The formula is as follows:

$$
x_{\text {norm }}=\frac{x-\min }{\max -\min }
$$

where $x_{n o r m}$ is the result of the normalization, $x$ is the value to be normalized, max is the upper value of the dataset and $\min$ is the smallest value of the dataset.

Heart Rate, Respiration Amplitude, Respiration Rate, Fixation Time and Blink Interval are the inputs of neural network. The value of the performance is the output. Because the duration of each flight is slightly different, the number of data in each dataset is slightly different, between 300 and 600 . The form of selected parameters and performance are all dimensionless and numerical. The selected parameters and the performance are shown in Table $\mathbf{1}$.

\subsection{Hybrid Model to Assess the Flight Performance}

In this section, we use MATLAB to establish the three different neural networks to assess and forecast the performance. For each run, the last 50 patterns are the forecasting samples and the former patterns are training samples. Data of each flight is run and get the results. 
Table 1. The illustration of physiological parameters and performance.

\begin{tabular}{|c|c|c|}
\hline Index & Unit & Expression \\
\hline \hline Heart Rate & Dimensionless & Numerical value \\
\hline Respiration Amplitude & Dimensionless & Numerical value \\
\hline Respiration Rate & Dimensionless & Numerical value \\
\hline Eye Blink Interval & Dimensionless & Numerical value \\
\hline Eye Fixation Time & Dimensionless & Numerical value \\
\hline Performance & Dimensionless & \\
\hline
\end{tabular}

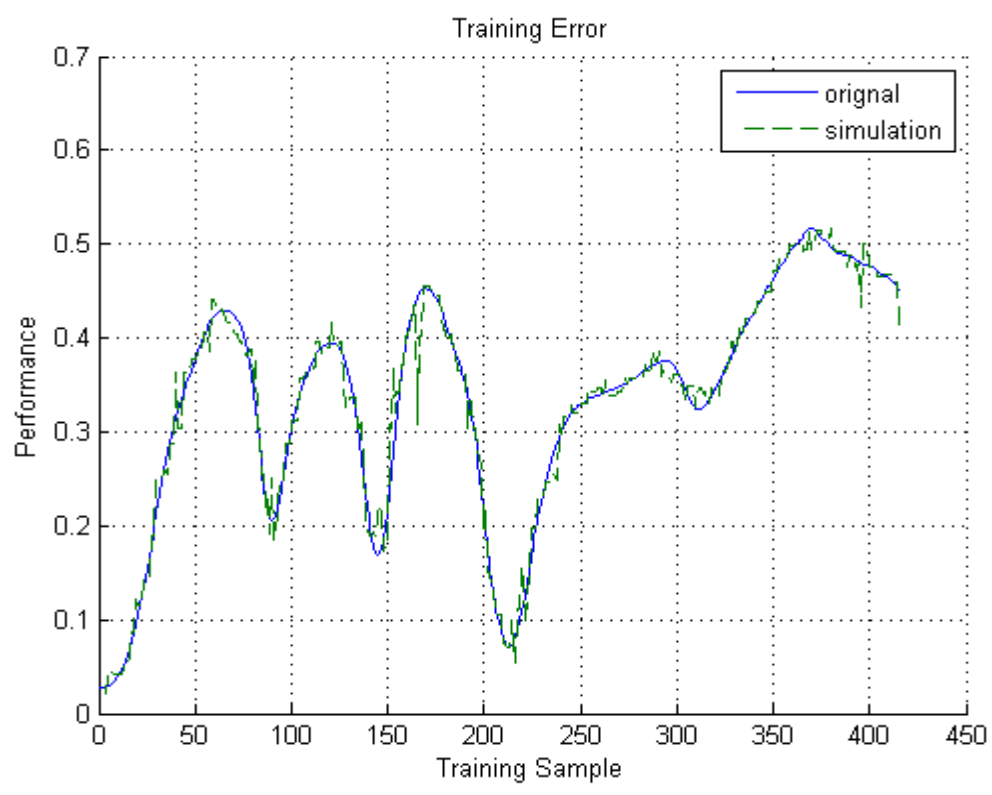

Fig. (6). The training error of CGA-Elman.

The first network is Elman neural network. It has 5 input layer neurons and 1 output layer neuron, which correspond to the five selected physiological parameters and one performance value. The number of hidden layer neurons is set as 15 by a series of trials, which verified that when the number of hidden layer is 15, the forecasting error of the Elman network is the minimum. The transfer function of hidden layer is "tansig" and the transfer function of output layer is "purelin". The second network is CGA-Elman. The GA initial parameters are set as following: population size: 80 ; evolution generation: 100; crossover rate: 0.3 ; mutation rate: 0.01 .Fig. (6) and Fig. (7) show the training result and forecasting results of one data. The trend of the change in the fitness value of each generation is shown in Fig. (8). It is obvious that the CGA-Elman is convergent. The relative error is $5.84 \%$. To verify the superiority and generalization of CGA-Elman, Elman, GA-Elman were used to run the same 20 sets of data. For the effective of the comparison, the initial parameters of GA-Elman are the same with that of CGA-Elman.
Key measurements (MAE, MAPE, MSE) are used to evaluate the forecasting capability of the three networks. Table 2 sets out the expressions of these indexes [16]. The comparison results of key measurements of the 20 datasets of the three networks are shown in Fig. (9). Fig. (9a-c) show the MAE, MAPE and MSE values respectively, which can be indicated that the prediction performance of CGA-Elman is vastly superior to GA-Elman and Elman. The average value of the key measurements of the 20 forecasting results and average training time of the four models are shown in Table 3.

\section{DISCUSSION}

As is shown in Table 3, all index values of CGA-Elman model are smaller than that of Elman and GA-Elman. The MAE of CGA-Elman is both reduced by $50 \%$ compared with Elman and about $13.5 \%$ compared with GA-Elman. The MAPE of CGA-Elman is both reduced by $69 \%$ compared with Elman and about 23\% compared with GA-Elman. The 


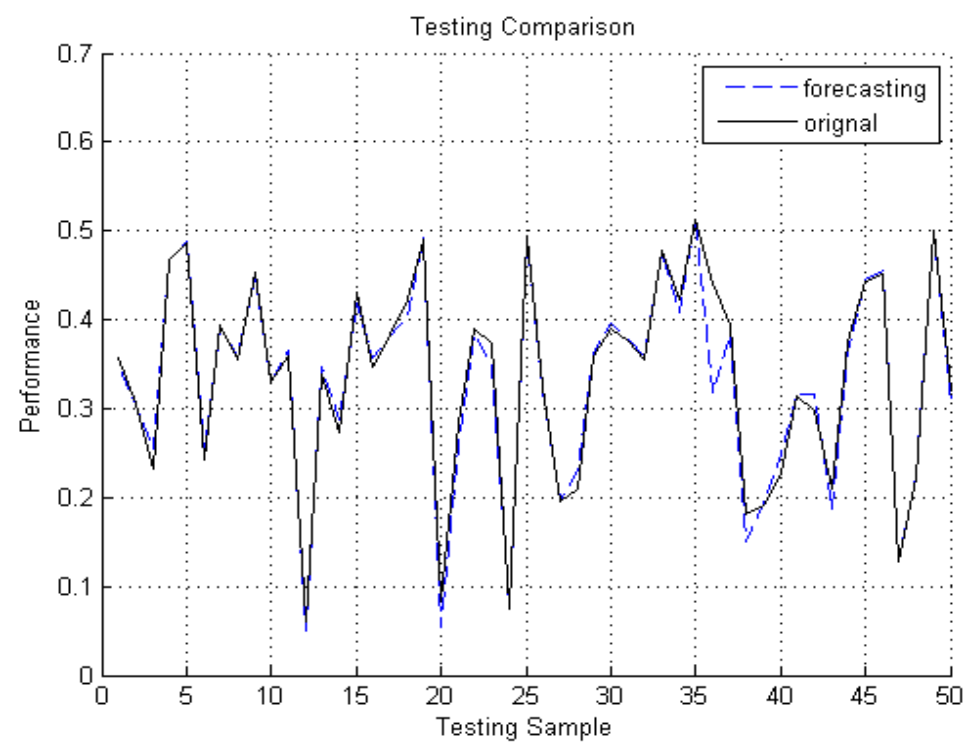

Fig. (7). The forecasting error of CGA-Elman.

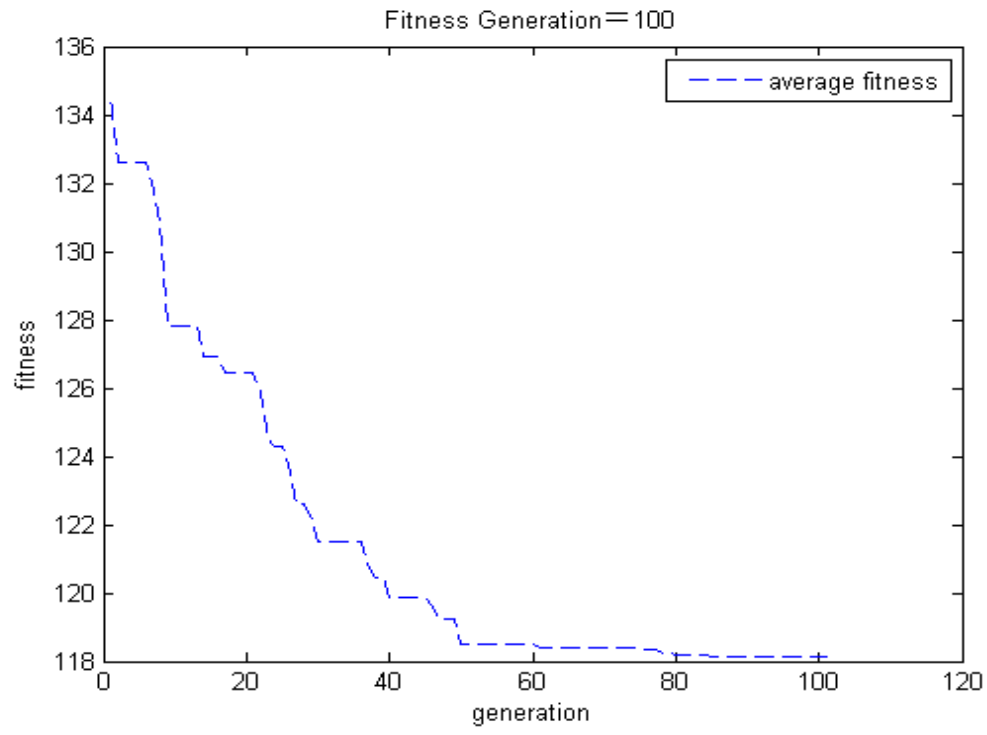

Fig. (8). The fitness degree of training samples of CGA-Elman.

Table 2. The expression of the error indexes.

\begin{tabular}{|c|c|c|}
\hline Index & Expression & Formula \\
\hline \hline MAE & Mean Absolute Error & $\sum_{t=1}^{n}\left|y_{t}-\hat{y}_{t}\right| / n$ \\
\hline MSE & Mean Squared Error & $\sum_{t=1}^{n}\left(y_{t}-\hat{y_{t}}\right)^{2} / n$ \\
\hline MAPE & Mean Absolute Percentage Error & $\left(100 \times \sum_{t=1}^{n}\left|\frac{y_{t}-\hat{y}_{t}}{y_{t}}\right|\right) / n$ \\
\hline
\end{tabular}




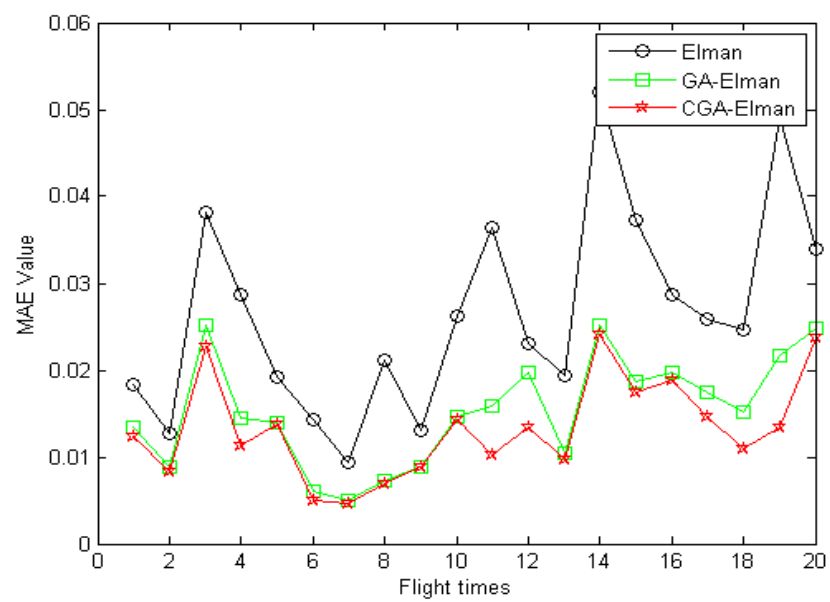

(a) MAE of the Three Networks

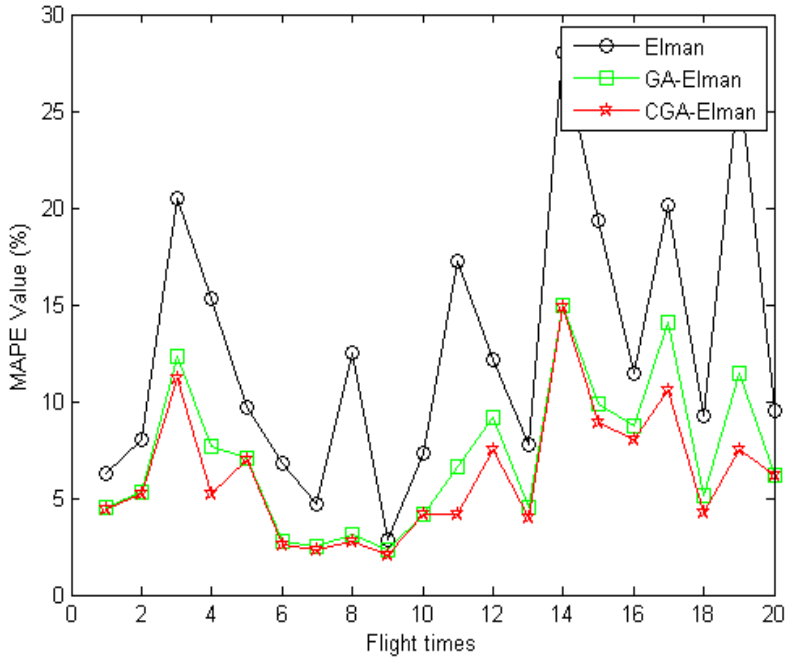

(b) MAPE of The Three Networks

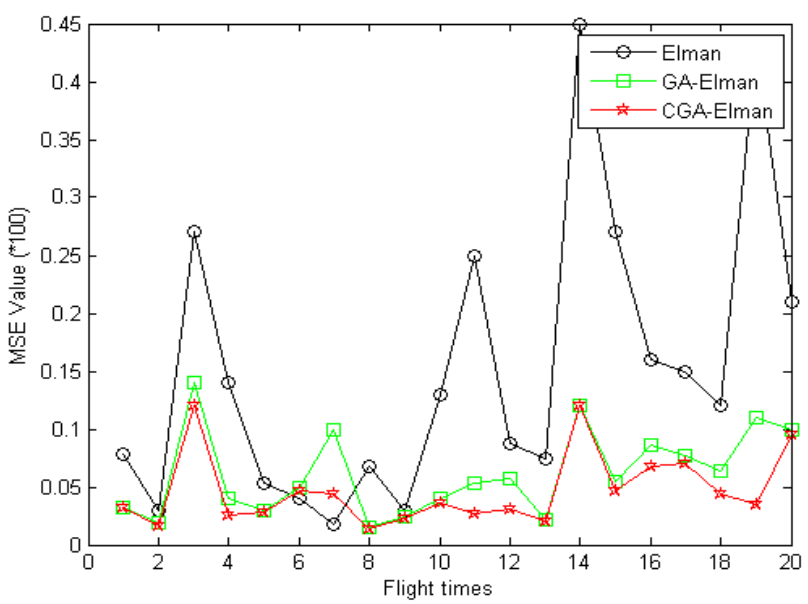

(c) MSE of the Three Networks

Fig. (9). The MAE, MAPE and MSE of the 20 sets of data based on the three models. 
Table 3. Comparison of average error analysis and average fitness of the three models.

\begin{tabular}{|c|c|c|c|c|}
\hline Model & MAE & MAPE & MSE(*100) & Fitness \\
\hline \hline Elman & 0.02656 & $12.77 \%$ & 0.1528 & No \\
\hline GA-Elman & 0.01532 & $7.12 \%$ & 0.0616 & 135.85 \\
\hline CGA-Elman & 0.01325 & $6.15 \%$ & 0.0473 & 110.82 \\
\hline
\end{tabular}

MSE of CGA-Elman is reduced by about $52 \%$ than Elman and by $13.6 \%$ than GA-Elman. The average fitness of CGAElman is $18.4 \%$ smaller than that of GA-Elman. It is concluded that the forecasting property of CGA-Elman neural network is better than the Elman and GA-Elman network. Considering the nonlinear influence of multi-dimensional data, it can be seen that CGA-Elman is more appropriate for assessing and forecasting the flight performance in this application. CGA-Elman does provide a new intelligence method for flight performance assessing and forecasting based on multiple physiological parameters.

\section{CONCLUSION}

In this paper, a new network called CGA-Elman is proposed to assess and forecast the flight performance based on multiple physiological signals. Compared with the other two forecasting models, the CGA-Elman model is presented to approximate the real performance value, generating better results. The CGA-Elman shows the superiority and effective in the matter of the forecasting of flight performance. It can be concluded that CGA-Elman network has the great application potential in the field of the assessment of the flight performance. The CGA-Elman optimized networks, to our best knowledge is for the first time being applied to assess and forecast flight performance in the scope of aviation, especially based on multiple physiological signals. This paper put forward a new kind of method for the assessing and forecasting of flight performance objectively, so there is a need for more efforts from researchers to develop more efficient method to solve this novel aviation problem.

\section{CONFLICT OF INTEREST}

The authors confirm that this article content has no conflict of interest.

\section{ACKNOWLEDGEMENTS}

This research work was supported by The National Basic Research Program of China (973 Program, 2010CB734103).

\section{REFERENCES}

[1] G. F. Wilson, "An analysis of mental workload in pilots during flight using multiple psychophysiological measures", Int. J. Avat. Psychol., vol.12, pp. 3-18, 2012

[2] S. Miyake, "Multivariate workload evaluation combining physiological and subjective measures", Int. J. Psychophysiol., vol. 40, pp. 233-238, 2001

[3] Y.H. Lee, and B.S. Liu, "Infligt workload assessment:comparison of subjective and physiology measurements", Aviat. Space Environm. Med., vol. 74, pp. 1078-1084, 2003.

[4] S. Miyake, "Multivariate workload evaluation combining physiological and subjective measures", Int. J. Psychophysiol., vol. 40, pp. $232-238,2001$.

[5] A. Sonderegger, and J. Sauer, "The influence of laboratory set-up in usability tests: effects on user performance,subjective ratings and physiological measures", Ergonomics, vol. 52, pp. 1350-1361, 2009.

[6] Y. Lee, and B. Liu, "Infligt workload assessment: comparison of subjective and physiology measurements", Aviat. Space Environ. Med., vol. 74, pp. 1078-1084, 2003.

[7] X. F. Yan, and D. Z. Chen, "Chaos-genetic algorithms for optimizing the operating conditions based on RBF-PLS model", Comput. Chem. Eng., vol. 27, pp. 1393-1404, 2003.

[8] W. Qi, "The hybrid forecasting model based on chaotic mapping, genetic algorithm and support vector machine", Expert Syst. Appl., vol. 37, pp. 1776-1783, 2001.

[9] X. Yuan, Y. Yuan, and Y. Zhang, "A hybrid chaotic genetic algorithm for short-term hydro system scheduling", Math. Comput Simul., vol. 59, pp. 319-327, 2002.

[10] J. Elman, "Finding structure in time", Cognit. Sci., vol. 14, pp. 179-211,1990

[11] S. Ding, Y. Zhang, and J. Chen, "Research on using genetic algorithms to optimize Elman neural networks", Neural, Comput, and Appl., vol. 23, pp. 293-297, 2013.

[12] Y. Kasahara, and Y. Yonezawa, "The properties of complex evolution in chaos generation process", In: Proc IEEE Int Conf Evol Comp, Nagoya, 1996, pp. 874-879.

[13] S. C. Leandro, "A quantum particle swarm optimizer with chaotic mutation operator", Chaos, Solut. Fract., vol. 37, pp. 1409-1418, 2008.

[14] R. May, "Simple mathematical models with very complicated dynamics", Nature, vol. 261, pp. 45-67, 1976.

[15] L. Ying, S. Fu, "Brief review on physiological and biochemical evaluations of human mental workload", Hum. Factor. Ergon. Man., vol. 22, pp. 177-187, 2012.

[16] Q. Wu, and R. Law, "A hybrid-forecasting model reducing Gaussian noise based on the Gaussian support vector regression machine and chaotic particle swarm optimization", Infor. Sci., vol. 238, pp. 96-110, 2013. (http://creativecommons.org/licenses/by-nc/3.0/) which permits unrestricted, non-commercial use, distribution and reproduction in any medium, provided the work is properly cited. 